
\title{
25 Research Square \\ Polygenic risk score for early prediction of sepsis risk in the polytrauma screening cohort
}

\section{Hongxiang Lu}

Army Medical University

\section{Dalin Wen}

Army Medical University

Jianhui Sun

Army Medical University

Juan Du

Army Meidcal University

Liang Qiao

Army Medical University

Huacai Zhang

Army Medical University

Ling Zeng

Army Medical University

Lianyang Zhang

Army Medical University

Jianxin Jiang

Army Medical University

Anqiang Zhang ( $\sim$ zhanganqiang@126.com)

State Key Laboratory of Trauma, Burns and Combined Injury, Institute of Surgery Research, Daping Hospital, Army Medical University https://orcid.org/0000-0002-0375-2078

\section{Research}

Keywords: Traumatic sepsis, Prediction, Weighted genetic risk score

Posted Date: February 20th, 2020

DOI: https://doi.org/10.21203/rs.2.24069/v1

License: (c) (i) This work is licensed under a Creative Commons Attribution 4.0 International License. Read Full License 
Version of Record: A version of this preprint was published at Frontiers in Genetics on November 12th, 2020. See the published version at https://doi.org/10.3389/fgene.2020.545564. 


\section{Abstract}

Background: Increasing genetic variants associated with sepsis have been identified by candidate-gene and genome-wide association studies, but single variant conferred minimal alterations in risk prediction. Our aimed to evaluate whether a weighted genetic risk score (wGRS) that aggregate information from multiple variants could improve risk discrimination of traumatic sepsis .

Methods: 64 genetic variants potential relating to sepsis were genotyped in Chinese trauma cohort. Genetic variants with mean decrease accuracy (MDA)>1.0 by random forest algorithms were selected to construct the multilocus wGRS. Area under curve (AUC) and Net reclassification improvement (NRI) were used to evaluate the discriminatory and reclassification ability of WGRS.

Results: Seventeen variants were extracted to construct the wGRS in 883 trauma patients. The wGRS was significantly associated with traumatic sepsis $(\mathrm{OR}=2.19,95 \% \mathrm{Cl}=1.53-3.15, \mathrm{P}=2.01 \times 10-5)$ after adjusted by age, sex, and ISS. Patients with higher wGRS have an increasing incidence of traumatic sepsis ( $P$ trend $=6.81 \times 10-8)$, higher SOFA $(P$ trend $=5.00 \times 10-3)$ and APACHEll score $(P$ trend $=1.00 \times 10-3)$. The AUC of risk prediction model incorporating wGRS into the clinical variables was $0.768(95 \% \mathrm{Cl}=0.739-0.796)$, with an increase of $3.40 \%(P=8.00 \times 10-4)$ versus clinical factors-only model. Furthermore, the NRI increased $25.18 \%(95 \% \mathrm{Cl}=17.84-32.51 \%)(\mathrm{P}=6.00 \times 10-5)$.

Conclusions: Our finding indicated that genetic predictors could improve the predictive ability of risk model for sepsis and highlighted the application among trauma populations, indicating that the sepsis risk assessment model will be a promising tool for high risk population screening and prediction.

\section{Background}

Trauma is the fourth leading cause of death around the world. Despite advances in clinical management of trauma patients, major trauma results in approximately $15 \%$ of disabilities and $10 \%$ of deaths [1]. One of the most serious complications post major trauma is the progressive dysfunction of vital organs, which are mainly caused by posttraumatic sepsis [2]. Sepsis and multiple organ dysfunction syndromes (MODS) compound the systemic inflammation triggered by the original injury and are difficult to distinguish [3]. Therefore, early prediction of sepsis and then providing the individual therapy accordingly are potential therapeutic managements of patients with traumatic sepsis [4].

Genetic variants of immune system modulate the host response to infection $[5,6]$. Therefore, whether genetic heterogeneity might have significant impact on sepsis development is an important question. Evidences from animal experiments and human genetic association studies demonstrated that genetic heterogeneity contributed to a significant portion of susceptibility to sepsis [7, 8]. In recent years, increasing numbers of sepsis predisposing variants have been identified by candidate gene and genomewide association studies (GWAS) [9, 10]. For example, rs4957796 within FER gene and rs5743551 located into the promoter region of TLR1 were associated with higher risk of sepsis [9, 11]. Moreover, rs 4919510 in MIR608 and rs2232618 in the coding region of LBP gene were both functional variants and conferred 
susceptibility to sepsis after trauma $[12,13]$. Therefore, delineating genetic heterogeneity for sepsis might contribute to the diagnostic approaches and therapeutic trials among trauma patients.

Due to the complex pathogenesis for development of sepsis, the prediction effect of individual genetic variant was relatively limited. Previous studies suggested great effect sizes might be obtained from genetic risk scores (GRS) comprising the cumulative effects of multiple genetic variants [14-16]. Therefore, we systematically reviewed all relevant studies and screened out the potential associated genetic variants with sepsis risk, and created a weight genetic risk score (wGRS) to evaluate the joint effect of multiple genetic variants for risk stratification of traumatic sepsis.

\section{Methods}

\section{Patient recruitment}

In the present study, 1000 major trauma patients were prospectively recruited. All patients were ethnic Han Chinese from the Department of Trauma Surgery in Daping Hospital between August, 2010 and March, 2016. Demographic and clinical data were collected from the electronic medical record and trauma biobank, as previously described [12]. Patients enrolled in the current study meet the following criteria: (1) aged between 18 and 65 years, (2) injury severity score (ISS) greater than 16; and (3) survival more than 48 hours in hospital. ISS was calculated according to the abbreviated injury score developed in 2005. As previously described, infection was considered to be a clinically obvious source or positive bacterial culture. Systemic inflammatory response syndrome (SIRS) was diagnosed based two or more of the following conditions: body temperature $<36^{\circ} \mathrm{C}$ or $>38^{\circ} \mathrm{C}$, heart rate $>90$ beats per min, respiratory rate $>20$ breaths per min, and white blood cell count $<4000 \mathrm{~mm}^{3}$ or $>12000 \mathrm{~mm}^{3}$ [17]. The diagnosis criterion of sepsis was based on the Sepsis 2.0 for patients who met at least two of SIRS criteria plus infection [18]. All patients were determined by two independent evaluators. Acute Physiology and Chronic Health Evaluation (APACHE) II and sequential organ failure assessment (SOFA) scores were applied to evaluate the severity and organ failures after injury during the hospital days. Candidate variants selection

Eligible studies were searched from PubMed, Embase, Medline, Web of Science, and HuGE databases before March 13, 2016 by using the following keywords: associated or synonymous with "sepsis" and "polymorphism". Furthermore, we reviewed the full text of each paper with the following criteria: 1) studies provided the number or frequency of genotypes in detail; and 2) these papers had an observational (case control or cohort) study design. The exclusion criteria were 1) studies with insufficient information; 2) abstract, comment, review and editorial; and 3) for duplicate publications, only the most recent or complete study was included. Finally, 316 articles investigating 333 variants involving 147 distinct genes were included in the study (Figure S1) [19]. Overall, we performed 334 meta-analyses on 65 variants with at least three study populations, and identified 16 variants significantly associated with the risk of sepsis. Of the other 268 variants with fewer than three populations, 48 variants were identified according to the reported association results. Finally, 64 candidate variants were selected for further genotyping and analyzing (Table S1). 


\section{Genotyping and quality control}

Blood specimens were obtained from trauma patients when admitted to the hospital within 24 hours. The genomic DNA was extracted from whole blood by a genomic DNA purification kit (Promega, Madison, WI, USA) according to the manufacturer's instructions. Genotyping was performed using the SNPscan method in all samples following the manufacturer's instructions [20]. One blank control in each plate was used for genotyping quality control, and $10 \%$ of samples were duplicated. The overall concordance rate was $100 \%$ among the duplicated samples. The genetic variants with calling rate of $>96 \%$, minor allele frequency (MAF) of $>0.01$, and Hardy-Weinberg equilibrium (HWE) at $P>0.01$ in the overall trauma cohort were included for further analysis. To calculate the wGRS, only the patients completely genotyped for all genetic variants were included for investigation.

\section{Statistical analysis}

Differences in categorical or continuous variables between subjects with sepsis and non-sepsis patients were assessed using Pearson's $\chi^{2}$ test or student $t$ test, respectively. Deviation from Hardy-Weinberg equilibrium (HWE) was tested using $\chi^{2}$ test. The association between individual variant and sepsis was determined by logistic regression analyses. The genotype of each variant was coded based on the amount of risk alleles, 0 for no risk alleles, 1 for heterozygous variant carriers and 2 for individuals carrying two risk alleles, respectively. Genetic variants with mean decrease accuracy (MDA) $>1$ by random forest algorithm were considered to have positive effects on the risk of sepsis and were chosen for construction of wGRS [21]. The wGRS was constructed on the base of the $\beta$ coefficients obtained from the logistic regression analysis in the additive model, and the equation was as follows: $w G R S=\beta 1 \times S N P 1+\beta 2 \times S N P 2+\ldots+\beta i \times S N P i$. The association between $w G R S$ and sepsis risk was analyzed by unconditional logistic regression. Meanwhile, the wGRS was divided into quartiles based on the distribution in the trauma cohort. Furthermore, the differences of SOFA and APACHEIl score were calculated among different quartiles of wGRS. Finally, the joint effect of the significant clinical variables and WGRS was assessed to predict the sepsis after trauma through multivariate logistic regression model. The discriminative ability of wGRS was evaluated by receiver-operator characteristic curves (ROC) and the $\mathrm{C}$ statistics. Net Reclassification Improvement (NRI) was applied to quantify the ability of correct reclassification after adding wGRS to clinical variables.

We used the variance inflation factor (VIF) for the collinearity diagnosis of the multivariate logistic regression. Then, a nomogram was constructed based on the multivariate logistic regression model incorporating the selected predictors. Decisioncurve analysis (DCA) was performed to determine the clinical usefulness of the nomogram by calculating the net benefits at different threshold probabilities in the trauma cohort.

All statistical analyses were performed with SPSS 17.0 and $R$ statistical software version 3.6.1. $P<0.05$ was considered as the criterion of statistical significance and all statistical tests were two sided. 


\section{Results}

\section{Clinical characteristics of trauma cohort}

Demographic data of 1000 trauma patients was summarized in Table S2. Patients were mostly young (mean age: $42.89 \pm 12.56$ years) and severely injured (mean ISS: $19.59 \pm 8.99$ ). Incidence of sepsis was $26.20 \%(n=262)$. Pneumonia and primary bloodstream infection was approximately $49.24 \%$ of all the documented infections. Gram-negative infections accounted for approximately $83.20 \%$, gram-positive infections for $6.87 \%$, and mixed gram-negative/gram-positive infections for $2.29 \%$ of sepsis patients. The median time for sepsis occurrence in the whole trauma cohort was $7.02 \pm 6.95$ days. The maximum of SOFA score and APACHE II score in hospital were $3.45 \pm 2.79$ and $8.27 \pm 6.01$, respectively. $21(2.10 \%)$ trauma patients died during the hospital days.

\section{Isolated variants have only a small impact on sepsis risk}

In the present study, 64 variants were successfully genotyped by the SNPscan method in 1000 trauma patients. The overall calling rate was greater than $96 \%$. All variants meet the criteria of MAF $>0.01$ and $P_{(H W E)}>0.01$ (Table S3). Due to genotyping failure in some samples, 883 patients with complete genotyping data for all 64 variants were finally selected for further analysis. We first tested the association between the 64 genetic variants individually and sepsis risk in unadjusted analyses applying an additive genetic model and using logistic regression analysis (Table S4). Unadjusted analyses showed that four variants were significantly associated with sepsis risk at a nominal level: rs2297518, located in the NOS2 gene $(\mathrm{OR}=1.53,95 \% \mathrm{Cl}=1.12-2.10, \mathrm{P}=0.01)$; rs10865710, located in the PPARG gene $(\mathrm{OR}=$ $1.32,95 \% \mathrm{Cl}=1.06-1.63, \mathrm{P}=0.01)$; rs740598, located in the HSPA12A gene $(\mathrm{OR}=1.25,95 \% \mathrm{Cl}=1.01-$ $1.53, P=0.04)$; and $r 55743551$, located in the TLR1 gene $(O R=1.26, P=0.04)$. The associations of the four variants with sepsis were confirmed using logistic regression analysis, adjusting for age and sex. None of the other variants was associated with sepsis. These results pointed to a relatively small impact of single variants on sepsis in our trauma cohort.

\section{A wGRS is significantly associated with traumatic sepsis}

To evaluate the joint effect of these genetic variants on sepsis risk, a random forest algorithm was developed. As shown in Table 1 and Fig. 1, a total of 17 genetic variants induced a positive effect (MDA > 1) by random forest algorithm (Table S4) and were selected in the subsequent calculation of the wGRS. For all trauma patients, the wGRS distribution was ranging from 0.68 to 3.69. The incidence of sepsis increased significantly along with the increase in number of risk alleles (Fig. 2A) and cases had more risk alleles than controls (Fig. 2B) using the wGRS of 17 variants $\left(P=3.47 \times 10^{-6}\right)$. As shown in Table S5, unadjusted logistic regression analyses confirmed the significant association between traumatic sepsis risk and wGRS $\left(\mathrm{OR}=2.42,95 \% \mathrm{Cl}=1.73-3.39, \mathrm{P}=3.03 \times 10^{-7}\right)$, which was also significantly associated with sepsis after adjusted by age, sex, and ISS through multivariable logistic regression analysis (OR = $2.19,95 \% \mathrm{Cl}=1.53-3.15, \mathrm{P}=2.01 \times 10^{-5}$ ). 
Table 1

17 selected genetic variants with MDA $>1$ by random forest algorithm.

\begin{tabular}{|lllllll|}
\hline Variant & Risk allele & Gene & MDA & Beta* & OR (95\%Cl) & P \\
\hline rs2297518 & G & NOS2 & 3.73 & 0.43 & $1.53(1.12-2.10)$ & 0.01 \\
\hline rs62375529 & C & FER & 2.89 & 0.29 & $1.34(0.87-2.05)$ & 0.19 \\
\hline rs760477 & A & TONSL & 2.70 & 0.03 & $1.03(0.83-1.28)$ & 0.97 \\
\hline rs10865710 & G & PPARG & 2.64 & 0.27 & $1.32(1.06-1.63)$ & 0.01 \\
\hline rs4957796 & C & FER & 2.25 & 0.29 & $1.34(0.87-2.05)$ & 0.19 \\
\hline rs1799983 & T & NOS3 & 2.08 & 0.22 & $1.24(0.93-1.70)$ & 0.18 \\
\hline rs11465996 & G & MD2 & 1.81 & 0.17 & $1.18(0.92-1.52)$ & 0.19 \\
\hline rs4919510 & C & SEMA4G & 1.56 & 0.05 & $1.05(0.85-1.31)$ & 0.63 \\
\hline rs2071746 & T & HMOX1 & 1.53 & 0.07 & $1.07(0.87-1.31)$ & 0.53 \\
\hline rs2069912 & C & PROC & 1.49 & 0.06 & $1.07(0.86-1.32)$ & 0.56 \\
\hline rs4073 & T & CXCL8 & 1.49 & 0.11 & $1.11(0.90-1.38)$ & 0.33 \\
\hline rs740598 & G & HSPA12A & 1.46 & 0.22 & $1.25(1.01-1.53)$ & 0.04 \\
\hline rs820336 & G & MYLK & 1.42 & 0.06 & $1.07(0.62-1.85)$ & 0.82 \\
\hline rs7851696 & T & FCN2 & 1.32 & 0.14 & $1.15(0.88-1.49)$ & 0.31 \\
\hline rs352162 & T & TLR9 & 1.30 & 0.12 & $1.13(0.91-1.41)$ & 0.27 \\
\hline rs5743867 & C & TOLLIP & 1.07 & 0.17 & $1.18(0.95-1.46)$ & 0.12 \\
\hline rs2243250 & C & IL4 & 1.03 & 0.08 & $1.08(0.83-1.40)$ & 0.57 \\
\hline *The beta coefficients column shows natural logarithms of the ORs; & Logistic regression in the \\
\hline additive model. & & & & & & \\
\hline
\end{tabular}

We further split the wGRS for sepsis into four subgroups according to its $25 \%$ percentage: low risk group (wGRS < 1.80), Medium risk group (wGRS $=1.80-2.20$ ), high risk group ( $w G R S=2.20-2.50$ ), and extremely high risk group (wGRS $\geq 2.50$ ). Based on the classification of the wGRS system, we found that in all the trauma cohort (883 individuals), 237 patients were classified into the low risk group with 42 (17.72\%) sepsis cases and 205 patients were classified into the extremely high risk group with 83 (40.48\%) sepsis cases. Compared with those individuals who had the lowest score (wGRS $<1.80$ ), the trauma patients with higher score had higher incidence of sepsis, with odds ratios of $1.47(95 \% \mathrm{Cl}=0.93-$ 2.30, $\mathrm{P}=0.10), 1.87\left(95 \% \mathrm{Cl}=1.20-2.92, \mathrm{P}=6.00 \times 10^{-3}\right)$, and $3.16\left(95 \% \mathrm{Cl}=2.05-4.88, \mathrm{P}=1.20 \times 10^{-7}\right)$, respectively $\left(P_{\text {trend }}=6.81 \times 10^{-8}\right)$ (Table 2$)$. Furthermore, we compared the SOFA score and APACHEII 
score in different wGRS subgroups, which also demonstrated higher SOFA score $\left(P_{\text {trend }}=5.00 \times 10^{-3}\right)$ and APACHEll score $\left(P_{\text {trend }}=1.00 \times 10^{-3}\right)$ were observed in patients with higher score, respectively (Table 3).

Table 2

Cumulative effects of WGRS on the risk of sepsis.

\begin{tabular}{|c|c|c|c|c|c|c|}
\hline wGRS quartile & $\begin{array}{l}\text { Patients, } \\
\mathrm{n}\end{array}$ & ISS & $\begin{array}{l}\text { Sepsis, } n \\
(\%)\end{array}$ & OR $(95 \% \mathrm{Cl})$ & $P_{\text {value }}$ & $P_{\text {trend }}$ \\
\hline$(0.70-1.80)(\leq \mathrm{Q} 25)$ & 237 & $\begin{array}{l}20.28 \pm \\
6.33\end{array}$ & $\begin{array}{l}42 \\
(17.72 \%)\end{array}$ & $\begin{array}{l}1.00 \\
\text { (reference) }\end{array}$ & & \\
\hline $\begin{array}{l}(1.80-2.20)(\mathrm{Q} 25 \sim \\
\text { Q50) }\end{array}$ & 225 & $\begin{array}{l}21.26 \pm \\
6.86\end{array}$ & $\begin{array}{l}54 \\
(24.00 \%)\end{array}$ & $\begin{array}{l}1.47(0.93- \\
2.30)\end{array}$ & 0.10 & \\
\hline $\begin{array}{l}(2.20-2.50)(\mathrm{Q} 50 \sim \\
\text { Q75) }\end{array}$ & 216 & $\begin{array}{l}21.11 \pm \\
7.57\end{array}$ & $\begin{array}{l}62 \\
(28.70 \%)\end{array}$ & $\begin{array}{l}1.87(1.20- \\
2.92)\end{array}$ & $\begin{array}{l}6.00 \times \\
10^{-3}\end{array}$ & \\
\hline$(2.50-3.70)(>Q 75)$ & 205 & $\begin{array}{l}21.22 \pm \\
8.04\end{array}$ & $\begin{array}{l}83 \\
(40.48 \%)\end{array}$ & $\begin{array}{l}3.16(2.05- \\
4.88)\end{array}$ & $\begin{array}{l}1.20 \times \\
10^{-7}\end{array}$ & $\begin{array}{l}6.81 \times \\
10^{-8}\end{array}$ \\
\hline
\end{tabular}

Table 3

Associations of WGRS with the severity and organ failure after trauma.

\begin{tabular}{|llll|}
\hline wGRS quartile & Patients, $\mathbf{n}$ & SOFA score & APACHEIl score* \\
\hline$(0.70-1.80)(\leq \mathrm{Q} 25)$ & 237 & $3.05 \pm 2.39$ & $7.12 \pm 5.23$ \\
\hline$(1.80-2.20)(\mathrm{Q} 25 \sim \mathrm{Q} 50)$ & 225 & $3.46 \pm 2.65$ & $8.38 \pm 6.40$ \\
\hline$(2.20-2.50)(\mathrm{Q} 50 \sim \mathrm{Q} 75)$ & 216 & $3.62 \pm 3.15$ & $8.65 \pm 6.61$ \\
\hline$(2.50-3.70)(>\mathrm{Q} 75)$ & 205 & $3.98 \pm 2.81$ & $9.41 \pm 5.86$ \\
\hline${ }^{*} \mathrm{P}_{\text {trend }}=5.00 \times 10^{-3}$ among groups; ${ }^{*} \mathrm{P}_{\text {trend }}=1.00 \times 10^{-3}$ among groups. \\
\hline
\end{tabular}

\section{Discriminative ability for traumatic sepsis}

The wGRS and ISS were identified as independent risk predictors of sepsis in trauma patients using the multivariate logistic regression algorithm (Table S5). Furthermore, the VIF of the two candidate predictors was 1.012, indicating that there was no collinearity. Therefore, wGRS and ISS were used to construct the prediction model of traumatic sepsis. To validate whether the wGRS could enhance the predictive value, we conducted ROC to evaluate the predictive ability of three models: only ISS, only wGRS, and ISS plus wGRS. The AUCs of only wGRS and only ISS were $0.619(95 \% \mathrm{Cl}=0.586-0.651)$ and $0.734(95 \% \mathrm{Cl}=$ 0.703-0.763), respectively. Our results demonstrated that when incorporating wGRS into the ISS, the AUC of the prediction model increased to $0.768(95 \% \mathrm{Cl}=0.739-0.796)$, with an increase of $3.40 \%(P=8.00 \times$ 
$10^{-4}$ ) (Fig. 3B). To confirm the improvement, we considered NRI to estimate the reclassification of the prediction model when wGRS was included. Compared with the ISS, these reclassification rates gave an estimated NRI of $25.18 \%$ by including the wGRS into the ISS $\left(95 \% \mathrm{Cl}=17.84-32.51 \%, \mathrm{P}=6.00 \times 10^{-5}\right)$ (Table 4). Therefore, when wGRS was added to the clinical model, the ability of the prediction model improved significantly.

Table 4

Reclassification of predicted risk with the addition of wGRS using NRI.

\begin{tabular}{|c|c|c|c|c|c|c|c|c|}
\hline \multirow[t]{2}{*}{$\begin{array}{l}\text { Predicted risk } \\
\text { (without wGRS) }\end{array}$} & \multicolumn{5}{|c|}{ Reclassified risk (with wGRS) } & \multicolumn{2}{|c|}{$\begin{array}{l}\mathrm{N}(\%) \text { of subjects } \\
\text { reclassified }\end{array}$} & \multirow[t]{2}{*}{$\begin{array}{l}\text { Net correctly } \\
\text { reclassified }\end{array}$} \\
\hline & $\begin{array}{l}< \\
5 \%\end{array}$ & $\begin{array}{l}5- \\
10 \%\end{array}$ & $\begin{array}{l}10- \\
15 \%\end{array}$ & $\begin{array}{l}15- \\
20 \%\end{array}$ & $\begin{array}{l}\geq \\
20 \%\end{array}$ & $\begin{array}{l}\text { Increased } \\
\text { risk }\end{array}$ & $\begin{array}{l}\text { Decreased } \\
\text { risk }\end{array}$ & \\
\hline \multicolumn{9}{|c|}{ Non-sepsis patients $(n=642)$} \\
\hline $0-5 \%$ & 0 & 0 & 0 & 0 & 0 & \multirow{5}{*}{$\begin{array}{l}74 \\
(11.53 \%)\end{array}$} & \multirow{5}{*}{$\begin{array}{l}217 \\
(33.80 \%)\end{array}$} & \multirow[t]{5}{*}{$22.27 \%$} \\
\hline $5-10 \%$ & 0 & 0 & 0 & 0 & 0 & & & \\
\hline $10-15 \%$ & 0 & 0 & 0 & 0 & 0 & & & \\
\hline $15-20 \%$ & 0 & 42 & 154 & 162 & 74 & & & \\
\hline$\geq 20 \%$ & 0 & 0 & 2 & 19 & 189 & & & \\
\hline \multicolumn{9}{|c|}{ Sepsis patients $(n=241)$} \\
\hline $0-5 \%$ & 0 & 0 & 0 & 0 & 0 & \multirow{5}{*}{$\begin{array}{l}26 \\
(10.79 \%)\end{array}$} & \multirow{5}{*}{$\begin{array}{l}19 \\
(7.88 \%)\end{array}$} & \multirow[t]{5}{*}{$2.91 \%$} \\
\hline $5-10 \%$ & 0 & 0 & 0 & 0 & 0 & & & \\
\hline $10-15 \%$ & 0 & 0 & 0 & 0 & 0 & & & \\
\hline $15-20 \%$ & 0 & 1 & 11 & 22 & 26 & & & \\
\hline$\geq 20 \%$ & 0 & 0 & 3 & 4 & 174 & & & \\
\hline \multicolumn{6}{|l|}{ NRI $(95 \% \mathrm{Cl})$} & \multicolumn{3}{|c|}{$25.18 \%(17.84-32.51 \%)$} \\
\hline \multicolumn{6}{|l|}{$P$} & \multicolumn{3}{|l|}{$6.00 \times 10^{-5}$} \\
\hline
\end{tabular}

\section{Clinical usefulness of the prediction model}

To provide an easy-to-use tool for clinicians, we constructed a nomogram incorporating the two predictors based on the multivariate logistic regression model that showed good calibration and discrimination in the trauma cohort (Fig. 3A, B). The AUC of the nomogram was 0.768 , suggesting that the nomogram achieved greater predictive efficacy than either the wGRS or the ISS alone. The DCA for the nomogram is presented in Fig. 3C. The DCA indicated that when the threshold probability for a doctor or a 
patient is within a range from 0 to 0.56 , the nomogram adds more net benefit than the "treat-all" or "treatnone" strategies, which indicated that the nomogram was clinically useful. For example, with a threshold probability of $40 \%$, use of the nomogram could provide an added net benefit of 0.052 compared to the "treat-all" or "treat-none" strategy.

\section{Discussion}

In the current study involving 883 trauma patients, 17 of 64 potential risk variants identified systematically by previously GWAS and candidate gene association studies were used to calculate weighted genetic risk score based on random forest algorithm. Risk assessment models incorporating the wGRS and ISS were a better tool to predict the risk value of traumatic sepsis. Our current study indicated that increased wGRS was significantly associated with higher risk of traumatic sepsis. The model with only the ISS shows low discriminatory accuracy (AUC $=0.734$ ). However, when we plus the wGRS based on 17 variants into the model, the AUC increases to $0.768\left(P=8.00 \times 10^{-4}\right)$, indicating that genetic predictors could improve the discriminatory ability of the traditional risk model.

For major trauma patients, identifying those at high risk of sepsis then initiating appropriate treatment would improve the intensive therapy and clinical management [3, 22-24]. Outcomes following major injury are affected by many factors, containing genetic variants, inflammatory response, immune dysfunction, coagulation dysfunction, tissue damage, and abnormal host responses to different pathogenic microorganisms. Recently, majority of detection scoring systems about sepsis focused on early and accurate sepsis diagnosis, such as Insight [25], SIRS, and SOFA, which were frequently changed during the outcome process of trauma. Many studies have indicated that genetic variants might be a major and stable factor for the prediction of sepsis risk. However, evidences also indicated that a single variant is not fully responsible for sepsis development [26, 27]. In the study, we genotyped 64 genetic variants previously identified as susceptibility loci for sepsis risk. Multiple candidate genes of those polymorphisms were involved in pattern recognition receptors (PRRs), signal molecules, transcription factor, cytokines, and other immune regulated genes. PRRs are essential for recognition of microbial components and damage-associated molecular patterns, and contribute to activation of the immune system $[28,29]$. Therefore, those genetic variants exhibited strong association with initiation and augmentation of sepsis [27, 30], such as the TLR1 -7202A/G (rs5743551) and TLR2 Asn248Ser (rs4833095) polymorphisms have affected the function of TLR genes, TNFA - 308G/A (rs1800629) and IL6 -572C/G (rs1800796) have affected the expression level of cytokine TNF-a and IL-6, respectively [31, 32]. Hence, genetic polymorphisms might be confirmed as potential beneficial biomarkers for evaluating sepsis risk in trauma patients. Furthermore, our data indicated these genetic biomarkers combined into the wGRS might improve the prediction accuracy.

To date, our study is the first attempt to construct and comprehensively evaluate the capacity of wGRS for risk prediction of traumatic sepsis. Despite previous studies have indicated genetic variants combined and/or into the traditional risk model could enhance the discriminatory capacity. For example, Jabandziev et al. [33] demonstrated specific combinations of five polymorphisms in the BPI (rs5743507), 
LBP (rs2232618), TLR4 (rs4986790), HSP70 (rs2227956), and IL-6 (rs1800795) genes appeared to predict outcome of life-threatening sepsis in children. Shimada's study [34] indicated the combined panel of TNFA - 308G/A and IL1B -31C/T plus APACHE II score might enable more accurate prediction of outcome in septic patients. Laurentiu et al. [8] summarized a few genetic variants observed in sepsis and suggested specific genetic polymorphisms could be applied for early prediction of sepsis incidence in the future. In our previous study [35], we also indicated eight functional polymorphisms (IL1B -1470, IL1B -511, IL1B -31, IL4 -589, IL6 -572, IL $8-251$, IL $10-819$, and TNFA - 308) could be combined together to predict the risk of sepsis and organ dysfunction after trauma. In the current study, we revealed the incidence of traumatic sepsis has been increased with the increasing of wGRS. Genomic variants combined into the wGRS could predict the risk of traumatic sepsis $(A U C=0.619)$, which was improved when plus the ISS factor (AUC = 0.768). To address the increasing discriminatory power, we studied the improved value of genetic factors to the clinical factor model by NRI [36]. The improvement in risk prediction of traumatic sepsis offered by wGRS was validated (Improved 25.18\%) by more detailed characterization and comparison between performances of models combined genetic variants plus ISS factor together.

This study has several notable strengths. Firstly, our study constructed the risk prediction model by the system of screening and evaluating genetic susceptibility from previous studies that has high predictive ability accuracy. Furthermore, genetic variants have several advantages as predictors, including remaining unchanged, predictable life-long risk and easy, accurate and cost-effective measurement [14, 37]. In addition, the combining of genetic and clinical factors into one model was feasible in clinical practice for trauma patients, which might improve the identification of patients at high risk for sepsis. However, some limitations should be acknowledged. Firstly, in our current study, only ISS was significantly different between sepsis and non-sepsis trauma patients and included into the prediction model, but other risk factors (antibiotic usage, blood transfusion, tracheal cannula et al.) could not be ignored in clinical practice [1], the prediction ability might be improved by adding these risk factors. Secondly, our sample size was relatively small and limited in Chinese population. Whether our findings could be extended to the general or other ethnic population needed to be determined. Thirdly, we did not take into account possible gene-environment interactions or gene-gene interactions, but many interactions exist in reality.

\section{Conclusions}

The current study investigates the risk predictive ability of accumulated genetic variants associated with traumatic sepsis in Chinese Han populations. The finding confirmed that trauma patients with a higher wGRS would be more susceptible to sepsis. When combined with other clinical factors, wGRS could improve the ability of personalized risk assessment for traumatic sepsis.

\section{Abbreviations}


ISS, injury severity score; MAF, minor allele frequency; HWE, Hardy-Weinberg equilibrium; MDA, mean decrease accuracy; wGRS, weight genetic risk score; SIRS, systemic inflammatory response syndrome; APACHE, Acute Physiology and Chronic Health Evaluation; SOFA, sequential organ failure assessment; MODS, multiple organ dysfunction syndrome; GWAS, genome wide association studies; AUC, area under the curve; ROC, receiver operating characteristic; AIC, Akaike's information criterion; NRI, net reclassification improvement.

\section{Declarations}

\section{Ethics approval and consent to participate}

Ethics approval for this study was obtained from the Ethical and Protocol Review Committees of Army Medical University. Informed written consent was obtained from the patients and their next of kin before enrollment, including explicit permission for DNA analysis and the collection of relevant clinical data. Patient confidentiality was preserved according to the guidelines of the Declaration of Helsinki.

\section{Consent for publication}

Not applicable

\section{Availability of data and materials}

The datasets used and/or analyzed during the current study are available from the corresponding author on reasonable request.

\section{Competing interests}

All authors declare that they have no competing interests.

\section{Funding}

This work is supported by National Natural Science Foundation of China $(81601677,81971830$, and 81571892), Science and Technology Innovation Project for Academicians of Chongqing (cstc2017jcyjyszXX0007), and Medical Research Funding of PLA of China (17QNP005 and AWS14C003). 
Conceptualization: Jianxin Jiang and Anqiang Zhang; Methodology: Hongxiang Lu, Jianhui Sun, Dalin Wen, Liang Qiao, and Huacai Zhang; Formal analysis and investigation: Hongxiang Lu, Dalin Wen, and Juan Du; Writing-original draft preparation: Hongxiang Lu and Dalin Wen; Writing-review and editing: Hongxiang Lu, Jianxin Jiang, and Anqiang Zhang; Funding acquisition: Jianxin Jiang, Ling Zeng, and Anqiang Zhang; Resources: Ling Zeng, Lianyang Zhang, and Dalin Wen; Supervision: Jianxin Jiang and Anqiang Zhang.

\section{Acknowledgements}

We warmly thank Prof. Min Wu, University of North Dakota, and Daolin Tang, University of Pittsburgh, for helpful discussions. Genesky Biotechnologies Inc., Shanghai, for genotyping of the samples and for technical support.

\section{References}

1. Lord JM, Midwinter MJ, Chen YF, Belli A, Brohi K, Kovacs EJ, Koenderman L, Kubes P, Lilford RJ: The systemic immune response to trauma: an overview of pathophysiology and treatment. Lancet 2014, 384(9952):1455-1465.

2. Park JH, Choi SH, Yoon YH, Park SJ, Kim JY, Cho HJ: Risk factors for sepsis in Korean trauma patients. European journal of trauma and emergency surgery : official publication of the European Trauma Society 2016, 42(4):453-458.

3. Lindner HA, Balaban U, Sturm T, Weiss C, Thiel M, Schneider-Lindner V: An Algorithm for Systemic Inflammatory Response Syndrome Criteria-Based Prediction of Sepsis in a Polytrauma Cohort. Critical care medicine 2016, 44(12):2199-2207.

4. Eriksson J, Gidlof A, Eriksson M, Larsson E, Brattstrom O, Oldner A: Thioredoxin a novel biomarker of post-injury sepsis. Free Radic Biol Med 2017, 104:138-143.

5. Eckert JK, Kim YJ, Kim JI, Gurtler K, Oh DY, Sur S, Lundvall L, Hamann L, van der Ploeg A, Pickkers P et al: The crystal structure of lipopolysaccharide binding protein reveals the location of a frequent mutation that impairs innate immunity. Immunity 2013, 39(4):647-660.

6. Hubacek JA, Stuber F, Frohlich D, Book M, Wetegrove S, Ritter M, Rothe G, Schmitz G: Gene variants of the bactericidal/permeability increasing protein and lipopolysaccharide binding protein in sepsis patients: gender-specific genetic predisposition to sepsis. Critical care medicine 2001, 29(3):557-561. 
7. Wurfel MM: Genetic insights into sepsis: what have we learned and how will it help? Curr Pharm Des 2008, 14(19):1900-1911.

8. David VL, Ercisli MF, Rogobete AF, Boia ES, Horhat R, Nitu R, Diaconu MM, Pirtea L, Ciuca I, Horhat D et al: Early Prediction of Sepsis Incidence in Critically III Patients Using Specific Genetic Polymorphisms. Biochemical genetics 2016.

9. Rautanen A, Mills TC, Gordon AC, Hutton P, Steffens M, Nuamah R, Chiche JD, Parks T, Chapman SJ, Davenport EE et al: Genome-wide association study of survival from sepsis due to pneumonia: an observational cohort study. The Lancet Respiratory medicine 2015, 3(1):53-60.

10. Villar J, Maca-Meyer N, Perez-Mendez L, Flores C: Bench-to-bedside review: Understanding genetic predisposition to sepsis. Crit Care 2004, 8(3):180-189.

11. Thompson CM, Holden TD, Rona G, Laxmanan B, Black RA, O'Keefe GE, Wurfel MM: Toll-like receptor 1 polymorphisms and associated outcomes in sepsis after traumatic injury: a candidate gene association study. Annals of surgery 2014, 259(1):179-185.

12. Zhang AQ, Gu W, Zeng L, Zhang LY, Du DY, Zhang M, Hao J, Yue CL, Jiang J: Genetic Variants of microRNA Sequences and Susceptibility to Sepsis in Patients With Major Blunt Trauma. Ann Surg 2015, 261(1):189-196.

13. Zeng L, Gu W, Zhang AQ, Zhang M, Zhang LY, Du DY, Huang SN, Jiang JX: A functional variant of lipopolysaccharide binding protein predisposes to sepsis and organ dysfunction in patients with major trauma. Ann Surg 2012, 255(1):147-157.

14. Jostins L, Barrett JC: Genetic risk prediction in complex disease. Human molecular genetics 2011, 20(R2):R182-188.

15. Chang J, Huang Y, Wei L, Ma B, Miao X, Li Y, Hu Z, Yu D, Jia W, Liu Y et al: Risk prediction of esophageal squamous-cell carcinoma with common genetic variants and lifestyle factors in Chinese population. Carcinogenesis 2013, 34(8):1782-1786.

16. Abraham G, Inouye M: Genomic risk prediction of complex human disease and its clinical application. Current opinion in genetics \& development 2015, 33:10-16.

17. Finkelsztein EJ, Jones DS, Ma KC, Pabon MA, Delgado T, Nakahira K, Arbo JE, Berlin DA, Schenck EJ, Choi AM et al: Comparison of qSOFA and SIRS for predicting adverse outcomes of patients with suspicion of sepsis outside the intensive care unit. Critical care 2017, 21(1):73.

18. Jacome T, Tatum D: Systemic Inflammatory Response Syndrome (SIRS) Score Independently Predicts Poor Outcome in Isolated Traumatic Brain Injury. Neurocrit Care 2018, 28(1):110-116.

19. Lu H, Wen D, Wang X, Gan L, Du J, Sun J, Zeng L, Jiang J, Zhang A: Host genetic variants in sepsis risk: a field synopsis and meta-analysis. Critical care 2019, 23(1):26.

20. Li H, Wang B, Liu D, Wang T, Li Q, Wang W, Li H: SNPscan as a high-performance screening tool for mutation hotspots of hearing loss-associated genes. Genomics 2015, 106(2):83-87.

21. Pisanu C, Preisig M, Castelao E, Glaus J, Pistis G, Squassina A, Del Zompo M, Merikangas KR, Waeber $G$, Vollenweider $P$ et al: A genetic risk score is differentially associated with migraine with 
and without aura. Hum Genet 2017, 136(8):999-1008. doi: 1010.1007/s00439-00017-01816-00435. Epub 02017 Jun 00427.

22. Taudien S, Lausser L, Giamarellos-Bourboulis EJ, Sponholz C, Schoneweck F, Felder M, Schirra LR, Schmid F, Gogos C, Groth S et al: Genetic Factors of the Disease Course After Sepsis: Rare Deleterious Variants Are Predictive. EBioMedicine 2016, 12:227-238.

23. Scherag A, Schoneweck F, Kesselmeier M, Taudien S, Platzer M, Felder M, Sponholz C, Rautanen A, Hill AVS, Hinds CJ et al: Genetic Factors of the Disease Course after Sepsis: A Genome-Wide Study for 28Day Mortality. EBioMedicine 2016, 12:239-246.

24. Brouwer MC, Read RC, van de Beek D: Host genetics and outcome in meningococcal disease: a systematic review and meta-analysis. Lancet Infect Dis 2010, 10(4):262-274.

25. Mao Q, Jay M, Hoffman JL, Calvert J, Barton C, Shimabukuro D, Shieh L, Chettipally U, Fletcher G, Kerem $Y$ et al: Multicentre validation of a sepsis prediction algorithm using only vital sign data in the emergency department, general ward and ICU. BMJ Open 2018, 8(1):e017833.

26. Bronkhorst MW, Patka P, Van Lieshout EM: Effects of Sequence Variations in Innate Immune Response Genes on Infectious Outcome in Trauma Patients: A Comprehensive Review. Shock 2015, 44(5):390-396.

27. Namath A, Patterson AJ: Genetic polymorphisms in sepsis. Crit Care Nurs Clin North Am 2011, 23(1):181-202.

28. Kawai T, Akira S: The role of pattern-recognition receptors in innate immunity: update on Toll-like receptors. Nat Immunol 2010, 11(5):373-384.

29. Wiersinga WJ, Leopold SJ, Cranendonk DR, van der Poll T: Host innate immune responses to sepsis. Virulence 2014, 5(1):36-44.

30. Arcaroli J, Fessler MB, Abraham E: Genetic polymorphisms and sepsis. Shock 2005, 24(4):300-312.

31. Duan ZX, Gu W, Zhang LY, Jiang DP, Zhou J, Du DY, Zen L, Chen KH, Liu Q, Jiang JX: Tumor necrosis factor alpha gene polymorphism is associated with the outcome of trauma patients in Chinese Han population. J Trauma 2011, 70(4):954-958.

32. Wurfel MM, Gordon AC, Holden TD, Radella F, Strout J, Kajikawa O, Ruzinski JT, Rona G, Black RA, Stratton $S$ et al: Toll-like receptor 1 polymorphisms affect innate immune responses and outcomes in sepsis. Am J Respir Crit Care Med 2008, 178(7):710-720.

33. Jabandziev P, Smerek M, Michalek J, Fedora M, Kosinova L, Hubacek JA, Michalek J: Multiple geneto-gene interactions in children with sepsis: a combination of five gene variants predicts outcome of life-threatening sepsis. Critical care 2014, 18(1):R1.

34. Shimada T, Oda S, Sadahiro T, Nakamura M, Hirayama Y, Watanabe E, Abe R, Nakada TA, Tateishi Y, Otani $S$ et al: Outcome prediction in sepsis combined use of genetic polymorphisms - A study in Japanese population. Cytokine 2011, 54(1):79-84.

35. Gu W, Zeng L, Zhou J, Jiang DP, Zhang L, Du DY, Hu P, Chen K, Liu Q, Wang ZG et al: Clinical relevance of 13 cytokine gene polymorphisms in Chinese major trauma patients. Intensive care medicine 2010, 36(7):1261-1265. 
36. Pencina MJ, D'Agostino RB, Sr., Steyerberg EW: Extensions of net reclassification improvement calculations to measure usefulness of new biomarkers. Stat Med 2011, 30(1):11-21.

37. Muller B, Wilcke A, Boulesteix AL, Brauer J, Passarge E, Boltze J, Kirsten H: Improved prediction of complex diseases by common genetic markers: state of the art and further perspectives. Hum Genet 2016, 135(3):259-272.

Figures

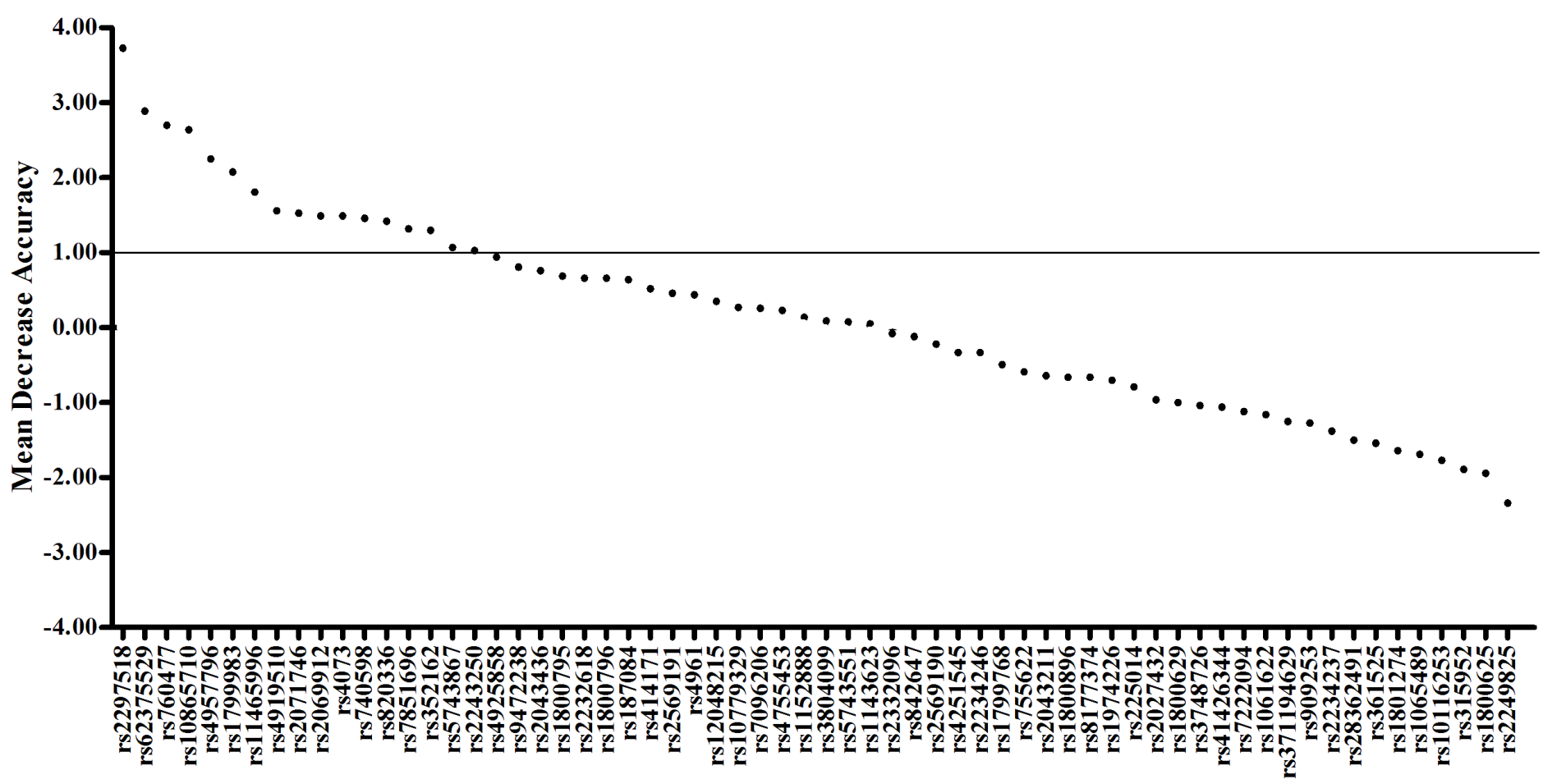

Figure 1

Random forest model including 17 variants previously associated with sepsis. The first 64 variables with the highest mean decrease accuracy are plotted. Seventeen sepsis-associated variants were shown to induce a positive change in mean decrease accuracy. These variants were thus considered to have a relevant influence on the model and were chosen for inclusion in the wGRS. 

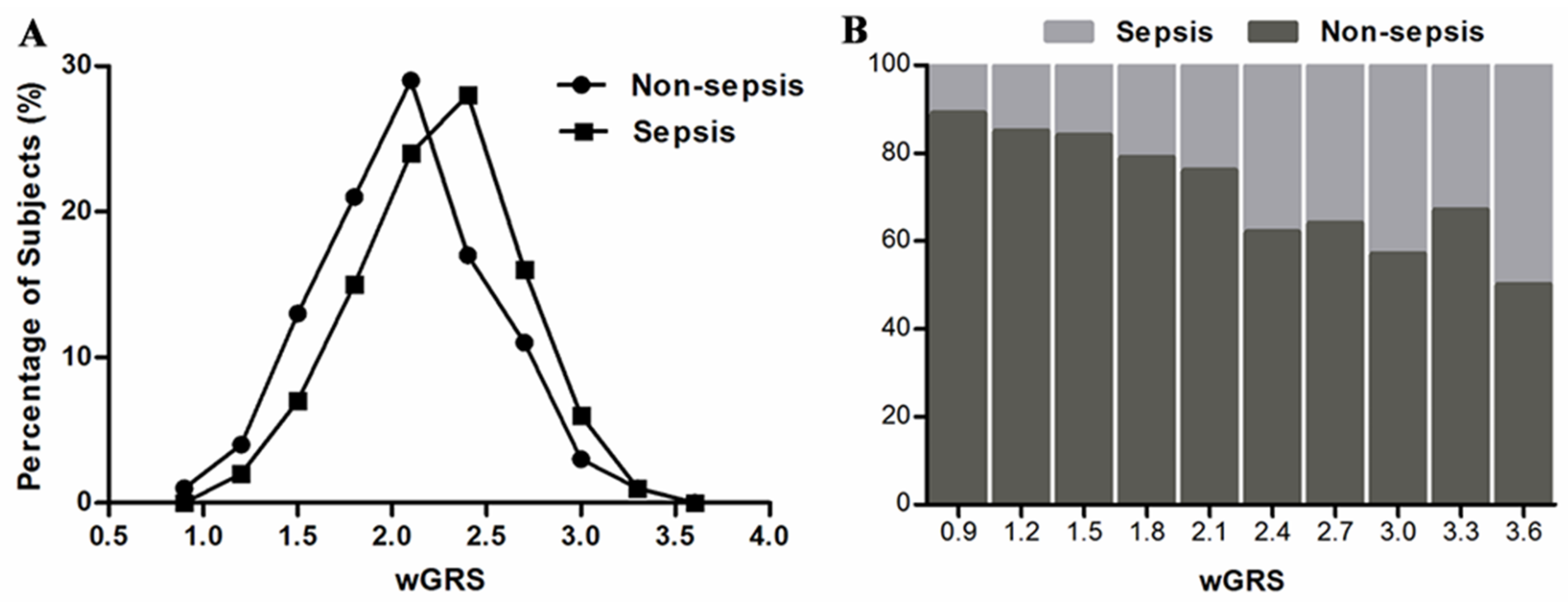

Figure 2

Distributions of the wGRS among sepsis cases and controls. A. The percentage of wGRS of 17 variants displaying significant difference among cases and controls. B. The distributions of wGRS of 17 variants among cases and controls. 
A

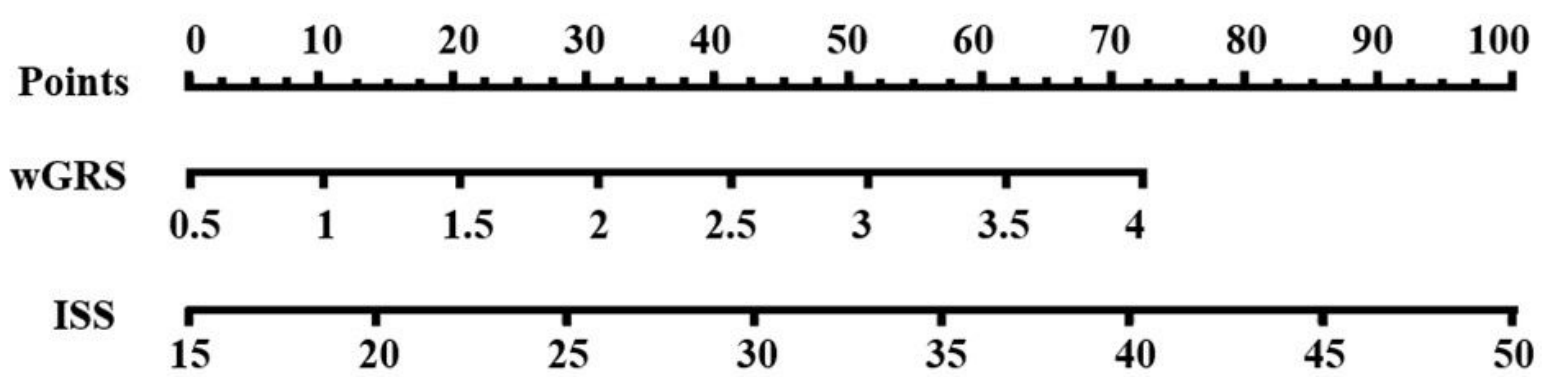

Total Points

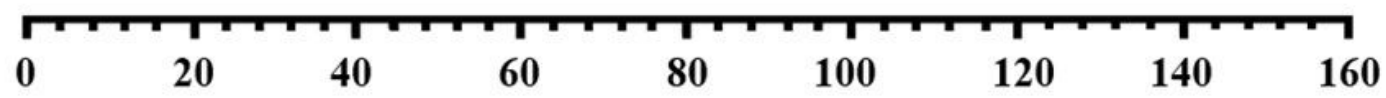

Risk of sepsis

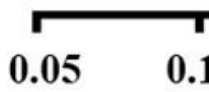

0.2

0.4

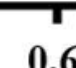

C

B

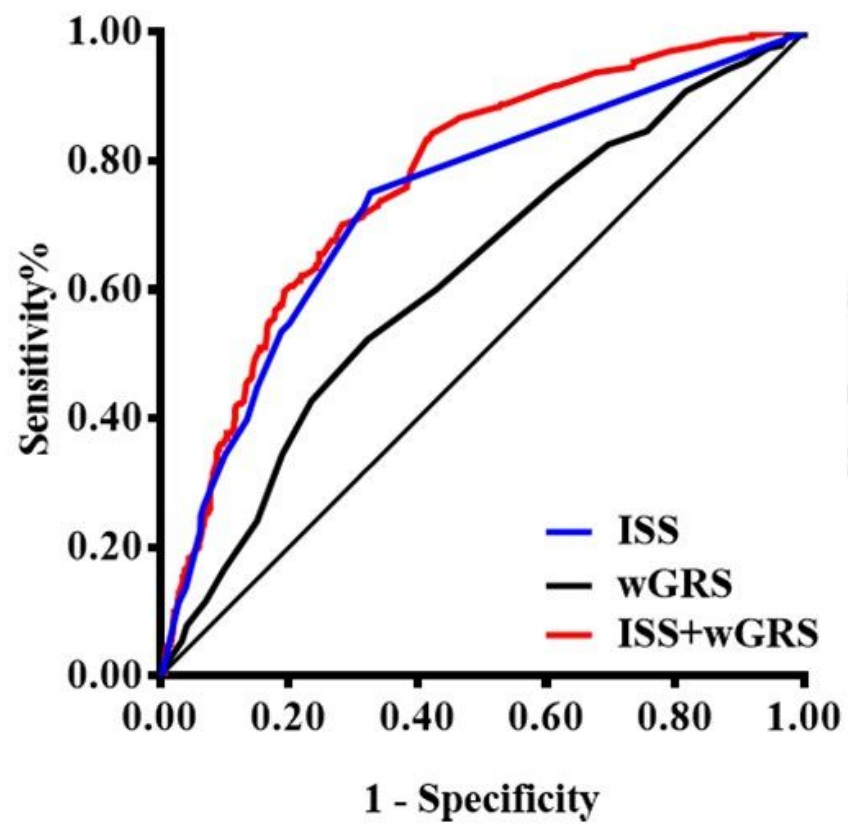

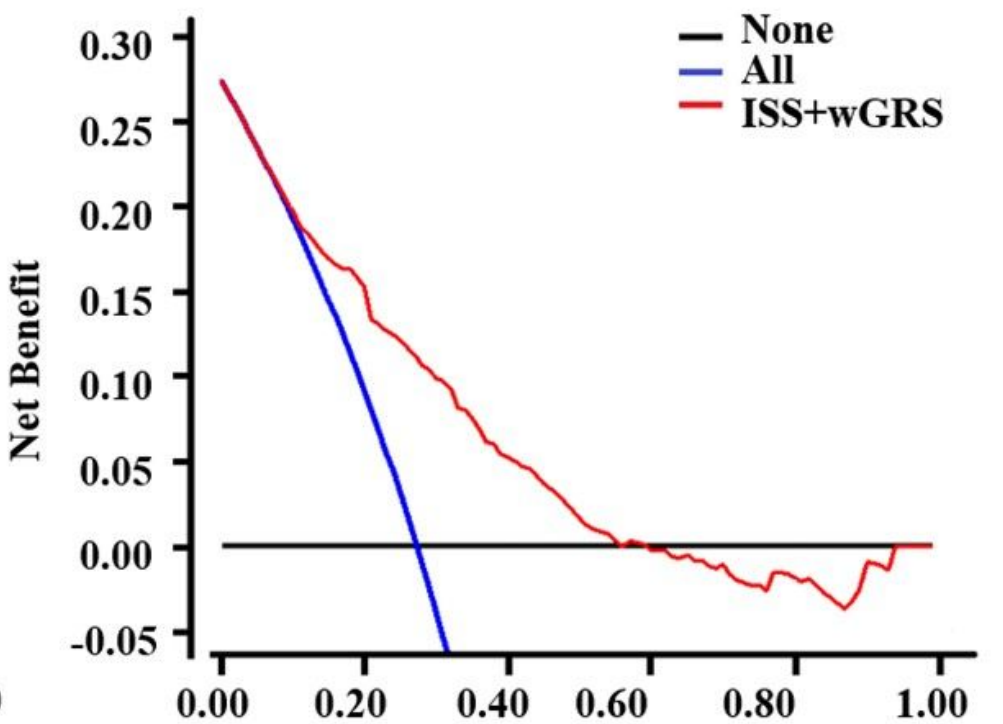

High Risk Threshold

Figure 3

Model comparisons and clinical usefulness of the nomogram. A. The nomogram incorporating ISS and wGRS was constructed for the prediction of sepsis after trauma. B. ROC curves of three models for sepsis risk. The area under the ROC curves (AUCs) are based on logistic regression models of only clinical risk factor (ISS), only genetic factor (wGRS), and both clinical risk factor and genetic factor (ISS+wGRS). C. DCA for the nomogram. The net benefit was plotted versus the threshold probability. The red line represents the nomogram. The gray and black lines represent the hypothesis that all patients and no patients had sepsis, respectively.

\section{Supplementary Files}

This is a list of supplementary files associated with this preprint. Click to download. 
- SupplementTables.docx

- Figures1.tif 